\title{
Human tissue-specific microenvironment: An essential requirement for mouse models of breast cancer
}

\author{
TIAN-SONG XIA $^{1 *}$, JUE WANG ${ }^{1 *}$, HONG YIN $^{2 *}$, QIANG DING ${ }^{1}$, YI-FEN ZHANG ${ }^{5}$, HAI-WEI YANG ${ }^{2}$, \\ XIAO-AN LIU ${ }^{1}$, MIN DONG ${ }^{6}$, QING DU ${ }^{3}$, LI-JUN LING ${ }^{1}$, XIAO-MING ZHA ${ }^{1}$, WEI FU ${ }^{1}$ and SHUI WANG ${ }^{1}$ \\ ${ }^{1}$ Department of Breast Surgery, ${ }^{2}$ Clinical Experiment Center, and ${ }^{3}$ Laboratory of General Surgery, \\ the First Affiliated Hospital of Nanjing Medical University; ${ }^{4}$ Department of Breast Surgery, Nanjing Maternity \\ and Child Health Care Hospital of Nanjing Medical University; ${ }^{5}$ Department of Pathology, Nanjing Drum Tower Hospital, \\ Nanjing University Medical School, Nanjing 210029; ${ }^{6}$ MR Division, Jiangsu Provincial Tumor Hospital, Jiangsu, P.R. China
}

Received March 12, 2010; Accepted April 29, 2010

DOI: $10.3892 /$ or_00000847

\begin{abstract}
An ideal mouse model should closely mimic a clinical situation. However, for most models available, this is not the case since clinical trials frequently fail to reproduce the highly encouraging therapeutic results obtained from preclinical studies performed using mouse models. In this study, in the process of extending the application of our previously established breast tissue-derived orthotopic and metastatic (BOM) model, the human breast cancer cell line MDA-MB231 failed to exhibit any osteotropic features that differed from previous studies. Our observations suggest that a human tissue-specific microenvironment could be an essential requirement for a successful mouse model of breast cancer. Here, multiple in vivo breast cancer models were used to confirm this hypothesis. Human breast tissue and cancellated bone were transplanted subcutaneously to female severe combined immunodeficiency disease (SCID) mice by different assemblies, to build several mouse models termed 'breast-breast', 'breastbone', 'bone-bone', 'MFP (mouse mammary fat pad)-bone', and 'MFP-breast' models. Two human breast cancer cell lines, MDA-MB-231 and MDA-MB-231BO, and the mouse breast cancer cell line TM40D were used. All cancer cells were labeled with GFP for gross observation. In addition, transplanted human tissues and various mouse tissues including bone, lung, liver, mesentery were examined microscopically. Based on morphological, immunohistochemical, and enzymohistochemical evidence obtained from several comparative
\end{abstract}

Correspondence to: Professor Shui Wang, Department of Breast Surgery, the First Affiliated Hospital of Nanjing Medical University, 300 Guangzhou Road, Nanjing 210029, P.R. China

E-mail:ws0801@gmail.com

*Contributed equally

Key words: human microenvironment, orthotope, metastasis, breast cancer, mouse model, xenograft experiments in 'breast-breast', 'breast-bone' and 'bone-bone' models, the BOM model was proved to be feasible and reliable. The organ tropism of the breast cancer cell line, which was derived from a mouse model by intracardiac inoculation in a pure mouse microenvironment, was reconsidered. The behavior of breast cancer cells in the mouse model was altered in response to the varying microenvironment. The results in this study suggest the human tissue-specific microenvironment is most likely an essential requirement in mouse models of breast cancer.

\section{Introduction}

According to the American Cancer Society, an estimated 192,370 new cases of breast cancer are expected to occur among women during 2009, and an estimated 40,170 deaths are expected (1). Breast cancer metastasis is an essentially incurable disease, and is associated with a poor prognosis. Recently, more attention has been given to the treatment of breast cancer metastasis. Mouse models offer an important platform for the evaluation of anti-cancer drugs/treatments and for investigation into the mechanisms involved in breast cancer metastasis. However, clinical practices frequently fail to reproduce highly encouraging therapeutic results obtained from prior preclinical studies performed using mouse models (2-5). This disparity between animal trials and clinical outcomes confers a possible disadvantage to using mouse models.

Continuing improvements have been made in mouse models of cancer. Complete immunodeficiency of the host mouse is now more achievable prior to tumor xenograft implantation. Additionally, human cell lines and metastatic variants are used extensively, organ-selective inoculation has been developed, and human tissues can now be implanted. However, even using genetically engineered mouse models (GEMM), there is little definitive or consistent evidence that these models show any advantages over traditional and economical transplant tumor models at preclinical trials (6-9). In our previous BOM model, we showed that the human organ-specific microenvironment can make breast cancer cells proliferate and metastasize more efficiently compared to the 
mouse microenvironment (10). The entire process of cancer metastasis is the following: growth and proliferation in the orthotopic site, detachment from the orthotopic site and invasion into the extracellular matrix (ECM), intravasation into the vessels, survival during circulation, extravasation from the vessels, and finally, growth and proliferation in the metastatic site $(11,12)$. The BOM model allows the complicated interactions between cancer cells and the microenvironment involved in each of the six steps to take place in human organ-specific conditions.

In order to mimic clinical bone metastasis, which occurs frequently in patients suffering from breast cancer and causes considerable complications and fatality, we implanted human bone and human breast tissue into SCID mice to establish another BOM model (breast-bone model). A well-known highly metastatic human breast cancer cell line MDA-MB231 was used in this model. In contrast to most traditional mouse models $(13,14)$, no metastasis was detected in either mouse bone or implanted human bone. Next, an osteotropic subclone, MDA-MB-231BO, derived from an intracardiac mouse model was used (13). Surprisingly, MDA-MB-231BO showed no different property in bone metastasis compared to MDA-MB-231. The two cell lines were then introduced to the 'bone-bone' model, in which human bone was implanted in both flanks and cells were inoculated around the implanted bone on the left side. Both cell lines grew well in the left implanted human bone of inoculating side, but failed to metastasize to the right implanted human bone or mouse bone. The results indicated that these breast cancer cell lines could grow and proliferate, rather than metastasize, in implanted human bone. Furthermore, in our previous 'breastbreast' model, MDA-MB-231 could metastasize from the left implanted human breast tissue to the right implanted human breast tissue. All of these results suggested that the ability of cells to grow in bone does not imply a tendency of metastasizing to bone. The osteotropism determined by traditional methods in the mouse microenvironment seemed to be unreliable. To further confirm these results, we introduced the two cell lines to the MFP-bone model. Additionally, a mouse breast cancer cell line TM40D was used. We found human breast cancer cells exhibited significantly higher tumorigenicity in orthotopic human tissues (breast and bone) compared to orthotopic mouse tissue (MFP), while TM40D showed lower tumorigenicity in orthotopic human tissues (breast) compared to MFP.

\section{Materials and methods}

Cell culture. The human breast cancer cell lines MDA-MB231 (ATCC) and MDA-MB-231BO (kindly provided by Dr J. Wang), and the mouse breast cancer cell line TM40D were cultured in Dulbecco's modified Eagle's medium (DMEM; Gibco, Grand Island, NY, USA), supplemented with $10 \%$ fetal bovine serum (FBS; Gibco) and 1\% penicillin-streptomycin solution (Gibco) in a humidified atmosphere of $5 \% \mathrm{CO}_{2}$ at $37^{\circ} \mathrm{C}$. All three cell lines were green fluorescent protein (GFP)-positive, as previously described (10).

Implantation of human tissues. Four- to six-week old female severe combined immunodeficient (SCID, CB-17IcrCrl-scid-
bgBR) mice were purchased from Model Animal Research Center of Nanjing University (MARC, Nanjing, Jiangsu Province, China). SCID mice were kept under specific pathogen free (SPF), temperature-controlled conditions $\left(20-24^{\circ} \mathrm{C}\right.$, humidity of $40-70 \%$ ). Cages, bedding, and drinking water were autoclaved and changed weekly. Food was sterilized by irradiation. All mice were maintained on a daily cycle of $12 \mathrm{~h}$ of light and $12 \mathrm{~h}$ of darkness.

Normal human breast tissues were obtained from freshly discarded material of elective breast reduction mammoplasty surgery. Female human bone tissues were obtained from discarded femoral heads from patients undergoing total hip replacement surgery. Sample collection was performed in accordance with the ethical guidelines of the Declaration of Helsinki, and approved by the ethics and research committee of the First Affiliated Hospital of Nanjing Medical University. Breast tissues were stripped of excess fat and sliced under sterile conditions into pieces $\sim 4 \mathrm{~mm}$ in width. Three pieces were selected randomly for histological examination to exclude primary malignant disease. Core bone was cut into fragments $\sim 4 \mathrm{~mm}$ in width following removal from the patient. The bone fragments were washed three times in $0-4 \%$ phosphate-buffered saline (PBS). Both kinds of human tissues were placed in ice cold PBS until implantation into SCID mice. Implantation was finished within $6 \mathrm{~h}$ after removal surgery. Prior to implantation, mice were anesthetized by intraperitoneal injection with $1 \%$ pentobarbital sodium (10 $\mu \mathrm{l} / \mathrm{g}$ body weight) (Sigma, Steinheim, Germany). Surgical procedures were performed as previously described, with some modifications (15-17). Briefly, 5-6 mm scalpel incisions were made in the skin of the mid-dorsal flank, through which five pieces of human breast tissue or three pieces of bone fragments were implanted subcutaneously. The mice received gentamycin in the drinking water $(800,000 \mathrm{U} / \mathrm{l})$ up to one week following the implantation. All mice studies were conducted according to the Guide for the Care and Use of Laboratory Animals and approved by the Animal Care and Use Committee of Nanjing Medical University.

In the 'breast-breast' model, human breast tissue was implanted in both the left and the right mid-dorsal flanks. In the 'breast-bone' model, human breast tissue was implanted in the left mid-dorsal flank and human bone was implanted in the right mid-dorsal flank. Breast tissue implantation was carried out three weeks after bone implantation. In the 'bonebone' model, human bone was implanted in both the left and the right mid-dorsal flanks. In MFP models, human bone or breast was implanted in the right mid-dorsal flank.

Injection of breast cancer cells into SCID mice. Breast cancer cells were grown to about $80 \%$ confluence and fed with fresh culture medium $24 \mathrm{~h}$ before inoculation. Cells were harvested using $0.25 \%$ trypsin and $0.02 \%$ disodium EDTA, washed in media, counted, and resuspended in PBS. One week after implantation of human breast tissue or four weeks after implantation of human bone, $5 \times 10^{5}$ breast cancer cells in $0.2 \mathrm{ml}$ PBS were inoculated into each mouse. Cancer cells were inoculated into the left human breast tissue in the 'breast-breast' and the 'breast-bone' models, adjacent to the left human bones in the 'bone-bone' model, and into leftinguina mouse MFP in MFP models. 
Gross observation of tumor formation, metastases, and sample collection. Eight weeks after inoculation of breast cancer cells, all mice were sacrificed and observed grossly by the Whole Body Imaging System (Illumatool 9900, Lightools Research, Encinitas, CA, USA). Three randomly chosen mice from the 'breast-bone' model with MDA-MB-231BO cells were used for magnetic resonance imaging (MRI Philips Achieva 1.5T, Sense Flex-Small soft coil. COR T1WI, TR 168 ms, TE 19 ms; T2WI, TR 1818 ms, TE 100 ms; slice thickness, $2 \mathrm{~mm}$, slice distance, $2 \mathrm{~mm}$. AX T1WI, TR $450 \mathrm{~ms}$, TE $15 \mathrm{~ms}$; T2WI, TR $1818 \mathrm{~ms}$, TE $100 \mathrm{~ms}$; slice thickness, $3 \mathrm{~mm}$, slice distance, $3 \mathrm{~mm}$ ). All implanted human tissues and mouse lung, mouse liver, mouse femur, and mouse soft tissues around the inoculation site were submitted for histological examination. Mouse spleens were isolated and triturated to a single cell suspension for FACS. Mouse blood was centrifuged at $2000 \mathrm{rpm}$ at $4^{\circ} \mathrm{C}$ for $10 \mathrm{~min}$ to extract serum and then used to assay human immunoglobulins by ELISA following the manufacturer's protocol (Bethyl, Montgomery, USA).

Histology, immunohistochemistry, and enzymohistochemistry. $H E$ staining. Specimens were fixed in $4 \%$ formalin (bones were then decalcified in 10\% EDTA for 48-72 h), dehydrated, and embedded in paraffin. Sections $\sim 5 \mu \mathrm{m}$ in thickness were stained with hematoxylin and eosin and examined under a microscope.

IHC. For immunohistochemical (IHC) staining, specimens were removed from paraffin and rehydrated with xylene and graded alcohols. Sections mounted on slides were subjected to antigen retrieval by a pressure cooker, and then incubated with monoclonal mouse antibodies specific for GFP (Invitrogen) or human CD34 (Santa Cruz Biotechnology). Immunocomplexes were visualized by the DAB method, and sections were counterstained with hematoxylin. Negative controls were serial sections that were processed without primary antibody. All histological analyses were performed by one pathologist.

Enzymohistochemical staining for TRAP (tartrate-resistant acid phosphatase). Dewaxed sections were preincubated for $20 \mathrm{~min}$ in buffer containing $50 \mathrm{mM}$ sodium acetate and $40 \mathrm{mM}$ sodium tartrate $(\mathrm{pH}$ 5.0). Sections were then incubated for $15 \mathrm{~min}$ at room temperature in the same buffer containing $2.5 \mathrm{mg} / \mathrm{ml}$ naphthol AS-MX phosphate (Sigma) in dimethylformamide as a substrate and $0.5 \mathrm{mg} / \mathrm{ml}$ fast garnetGBC (Sigma) as a color indicator for the reaction product. After washing with distilled water, the sections were counterstained with methyl green and mounted in Kaiser's glycerol jelly.

Fluorescence-activated cell sorting (FACS) analysis. FACS analysis was conducted on the entire spleen removed from normal adult female SCID mice, or mice harboring human bone fragments at 12 weeks post-implantation. Spleen cells were isolated by dissociating the tissue with the plunger end of a 5-ml syringe and then passing the cells through a $75-\mu \mathrm{m}$ cell culture filter. Cells were resuspended in PBS supplemented with $1 \%$ fetal bovine serum. One million cells were incubated with PerCP-Cy5.5-conjugated anti-human CD19 mAb or PerCP-Cy5.5-conjugated isotype control (BD
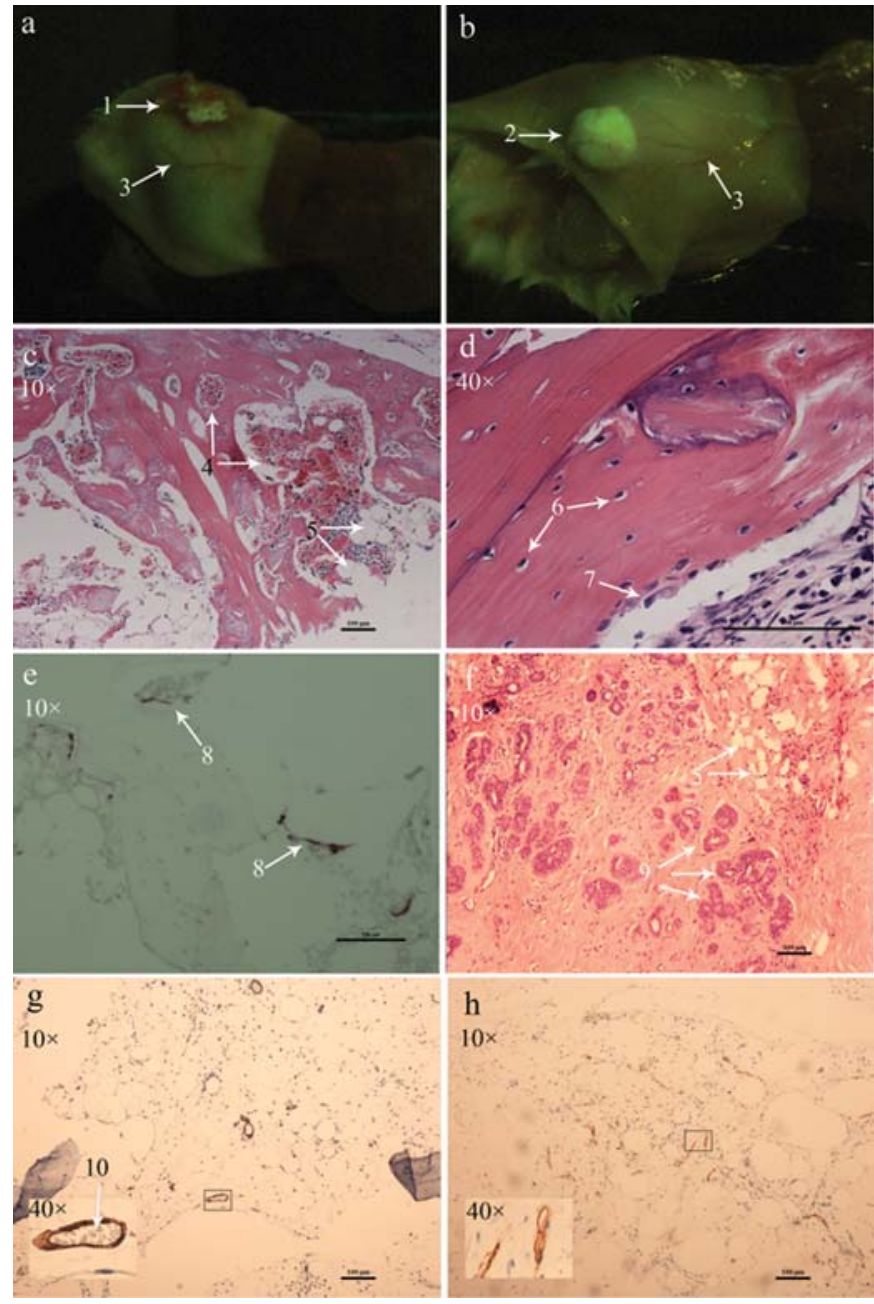

$\mathrm{h}$

$10 \times$

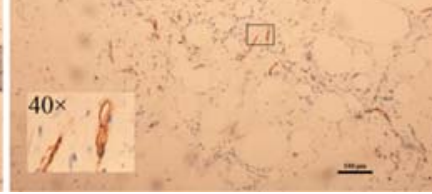

Figure 1. Gross observation and histological examination of implanted human tissues. Neoangiogenesis of mouse blood vessels supported implanted human tissues. (a) The gross observation of implanted human bone. (b) The gross observation of implanted human breast tissue. 1, The implanted human bone; 2 , the implanted human breast; 3 , the neoangiogenesis of mouse blood vessels supporting human implants. The structure and cells were remained in implanted human tissues. (c) The bone architecture of implanted human bone (H\&E staining). (d) The bone cells (H\&E staining). (e) TRAP staining of implanted human bone. (f) H\&E staining of the breast lobular architecture in implanted human breast. 4 , The marrow spaces; 5 , the adipocytes; 6 , the osteocytes; 7 , the osteoblasts; 8 , the osteoclasts; 9 , the lobular architecture in the implanted human breast tissues. Human derived blood vessels in implanted tissues were alive and functional. (g) Anti-human CD34 staining of implanted human bone. (h) Anti-human CD34 staining of implanted human breast tissue. 10, The red blood cells in blood vessel. Scale, $100 \mu \mathrm{m}$ (c, d, e, f, g and h). Magnifications x10, x40.

PharMingen, San Jose, CA, USA) at a final concentration of $10 \mu \mathrm{g} / \mathrm{ml}$ at $37^{\circ} \mathrm{C}$ for $1 \mathrm{~h}$. After two washes with $1 \% \mathrm{BSA} /$ PBS, cells were finally resuspended in PBS and analyzed on a FACSAria flow cytometer with the CellDiva software (BD Biosciences).

Statistical analysis. Fisher's exact test was applied using SPSS statistical software and $\mathrm{p}<0.05$ was taken as the statistically significant difference.

\section{Results}

Functional viability of implanted human tissues in mice. Neoangiogenesis of mouse blood vessels supporting implanted 

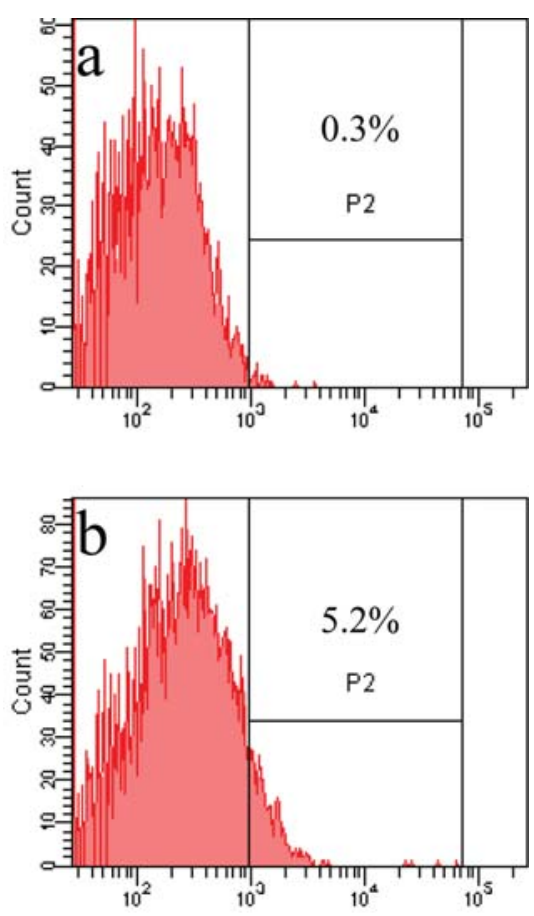

Figure 2. FACS analysis of human B cells from mouse spleen. Cells were isolated from the spleen of SCID mice not implanted with human bone (control) or mice ('bone-bone', 'breast-bone', 'MFP-bone') implanted with human bone 12 weeks post transplantation. Single cell suspensions were labeled with antibodies specific for human CD19-FTIC. (a) Cells isolated from the spleens of SCID mice not implanted with human bone. (b) Cells isolated from the spleens of SCID mice implanted with human bone. human bone (Fig. 1a) and human breast tissue (Fig. 1b) was observed. Bone architecture and stromal cells (Fig. 1c), osteocytes, osteoblasts (Fig. 1d), osteoclasts (Fig. 1e), were clearly present in the implanted human bone. Glandular tube and lobular architecture were observed in the implanted human breast tissue (Fig. 1f). Additionally, a wide variety of the normal breast cell types were clearly present in the implanted human breast (Fig. 1f). Human-derived blood vessels were still reserved in both implanted bone (Fig. 1g) and breast tissue (Fig. 1h) stained positive for CD34 using a human specific antibody to CD34. Mouse serum from mice with human bone was subjected to ELISA, which showed that human $\operatorname{IgG}$ was present at levels of $118.7 \pm 20.4 \mu \mathrm{g} / \mathrm{ml}$. Flow cytometry was performed on single-cell preparations of whole spleens from mice implanted with human bone using the human CD19 marker which is specific for human B cells. CD19 positive cells were not detected in spleen preparations from control mice devoid of human bone. In contrast, over $4 \%$ of the cells from the spleen of mice implanted with human bone were human CD19 positive (Fig. 2). Together, these data indicated that the human tissue implants were viable in vivo.

Tumor formation following inoculation of different breast cancer cells in mouse models. MDA-MB-231 cells grew well in orthotopic human breast tissue in both the 'breast-breast' and 'breast-bone' models. These cells could metastasize to human breast tissue but not human bone in the right side (Table I). No metastasis was detected in human bone or mouse

Table I. Tumor formation of MDA-MB-231 in 'breast-breast' model compared with 'breast-bone' model.

\begin{tabular}{|c|c|c|c|c|c|c|c|}
\hline & Lf-Br & $\mathrm{Rt}-\mathrm{Br} / \mathrm{Bo}$ & Ms-Bo & Mes & Ms-Lv & Ms-Lu & Bl-as \\
\hline $\mathrm{Br}-\mathrm{Br}$ & $9 / 9$ & $5 / 9^{a}$ & $0 / 9$ & $4 / 9$ & $0 / 9$ & $0 / 9$ & $0 / 9$ \\
\hline Br-Bo & $9 / 9$ & $0 / 9$ & $0 / 9$ & $4 / 9$ & $1 / 9$ & $0 / 9$ & $0 / 9$ \\
\hline
\end{tabular}

MDA-MB-231 cells were detected in all orthotopic human breast tissue in both models. They metastasized to distant human breast tissue but not human bone. Mouse tissues were rarely involved except mesentery. N/M, breast cancer cells were detected in corresponding tissue in $\mathrm{N}$ of M mice. Br-Br, 'breast-breast' model; Br-Bo, 'breast-bone' model; Lf-Br, implanted human breast tissue in left flank (inoculating site); Rt-Br, implanted human breast tissue in right flank; Rt-Bo, implanted human bone in right flank; Ms-Bo, mouse bone; Mes, mouse mesentery; Ms-Lv, mouse liver; Ms-Lu, mouse lung. Bl-as, bloody ascites. ${ }^{a} \mathrm{p}<0.05$, 'breast-breast' model compared with 'breast-bone' model.

Table II. Tumor formation of MDA-MB-231BO compared with MDA-MB-231 in 'breast-bone' model.

\begin{tabular}{|c|c|c|c|c|c|c|c|}
\hline Br-Bo & $\mathrm{Lf}-\mathrm{Br}$ & Rt-Bo & Ms-Bo & Mes & Ms-Lv & Ms-Lu & Bl-as \\
\hline 231BO & $9 / 9$ & $0 / 9$ & $0 / 9$ & $3 / 9$ & $0 / 9$ & $0 / 9$ & $0 / 9$ \\
\hline 231 & $9 / 9$ & $0 / 9$ & $0 / 9$ & $4 / 9$ & $1 / 9$ & $0 / 9$ & $0 / 9$ \\
\hline
\end{tabular}

The two cell lines were detected in all orthotopic human breast tissue in 'breast-bone' model. However, they did not metastasize to distant human and mouse bone. N/M, breast cancer cells were detected in corresponding tissue in $\mathrm{N}$ of M mice. Br-Bo, 'breast-bone' model; Lf-Br, implanted human breast tissue in left flank (inoculating site); Rt-Bo, implanted human bone in right flank; Ms-Bo, mouse bone; Mes, mouse mesentery; Ms-Lv, mouse liver; Ms-Lu, mouse lung; Bl-as, bloody ascites; 231BO, MDA-MB-231BO; 231, MDA-MB-231. 

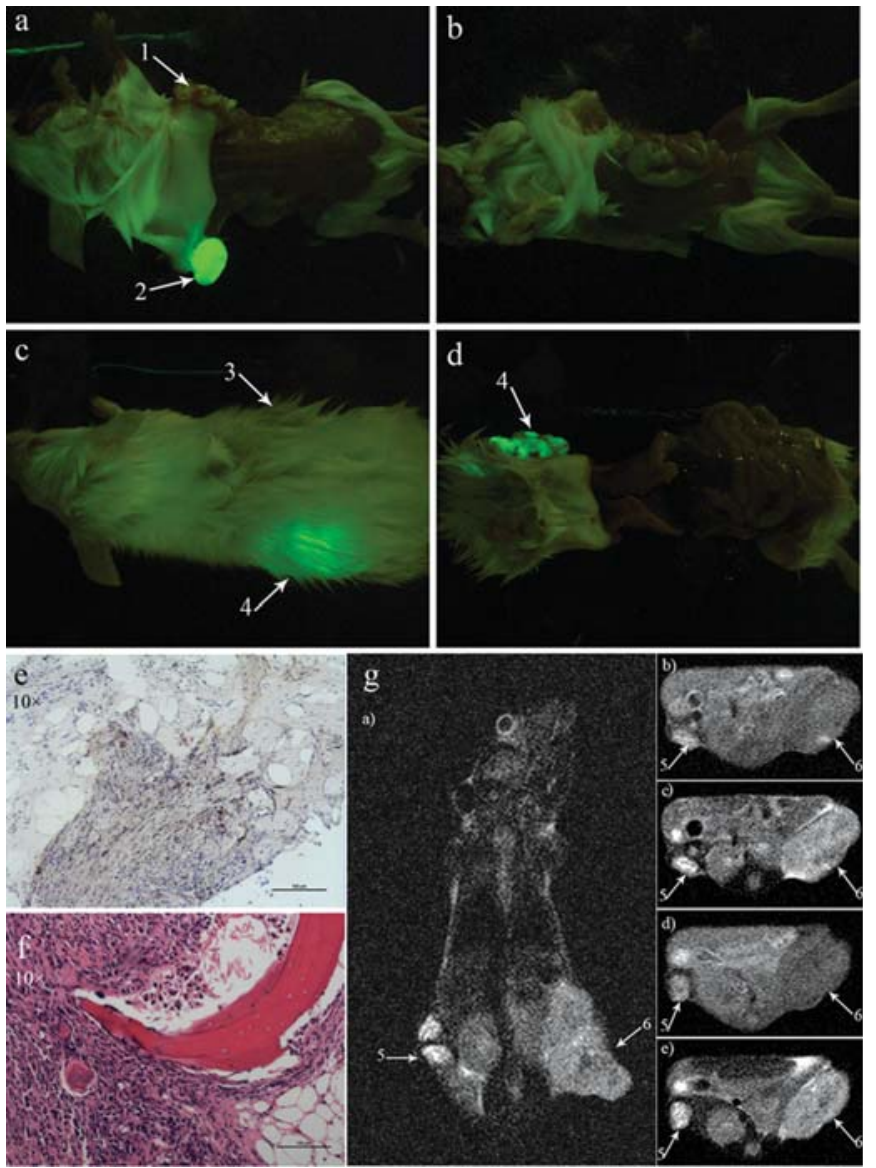

Figure 3. Gross observation of the MDA-MB-231BO tumors in the 'bonebone'/'breast-bone' model group and histological examination of the human bone inoculating-site. MDA-MB-231 cells formed extremely large tumor in orthotopic human breast in the 'breast-bone' model and grew well in inoculated human bone (left side) in the 'bone-bone' model but could not metastasize to human bone in the right side or mouse bone. (a) Fluorescent gross observation of the 'bone-bone' model (prone position) with MDAMB-231BO cells. (b) Fluorescent gross observation of the 'bone-bone' model (dorsal position) with MDA-MB-231BO cells. (c) Fluorescent gross observation of the 'breast-bone' model (prone position) with MDA-MB 231BO cells. (d) Fluorescent gross observation of the 'breast-bone' model (dorsal position) with MDA-MB-231BO cells. (e) Positive anti-GFP staining of orthotopic tumors in implanted human bone. (f) The H\&E staining of orthotopic tumors in implanted human bone. (g) MRI imaging of the "breastbone' model inoculated with MDA-MB-231BO cells. (a) The coronal-view image of T2WI. b), c), d) and e) Images of axial view. b) and c) present the same deck; d) and e) present the same deck. R, right side. b) and d) are T1WI while c) and e) are T2WI. The tumor presents a high signal in T2WI, but a low signal in T1WI. The bones present a high signal in both conditions. 1, The fluorescent negative implanted human bone in the noninoculated site of the 'bone-bone' model; 2, the fluorescent tumor in the inoculated site of implanted human bone of the 'bone-bone' model; 3 , the fluorescent negative implanted human bone in the right flank of the 'breastbone' model; 4 , the fluorescent orthotopic tumor in implanted human breast tissue; 5 , the implanted human bone; 6 , the tumor. Scale, $100 \mu \mathrm{m}$ (e and $\mathrm{f}$ ). Magnification x 10 .

bone in the 'breast-bone' model following inoculation with either MDA-MB-231BO or MDA-MB-231 cells (Table II). MDA-MB-231BO cells, however, grew much faster and were larger (Fig. 3c, d and g) in orthotopic human breast tissue compared to MDA-MB-231 (data not shown). Both MDA-MB-231BO and MDA-MB-231 cells grew well in orthotopic human bone in the 'bone-bone' model but did not metastasize to human bone in the right side or mouse bone

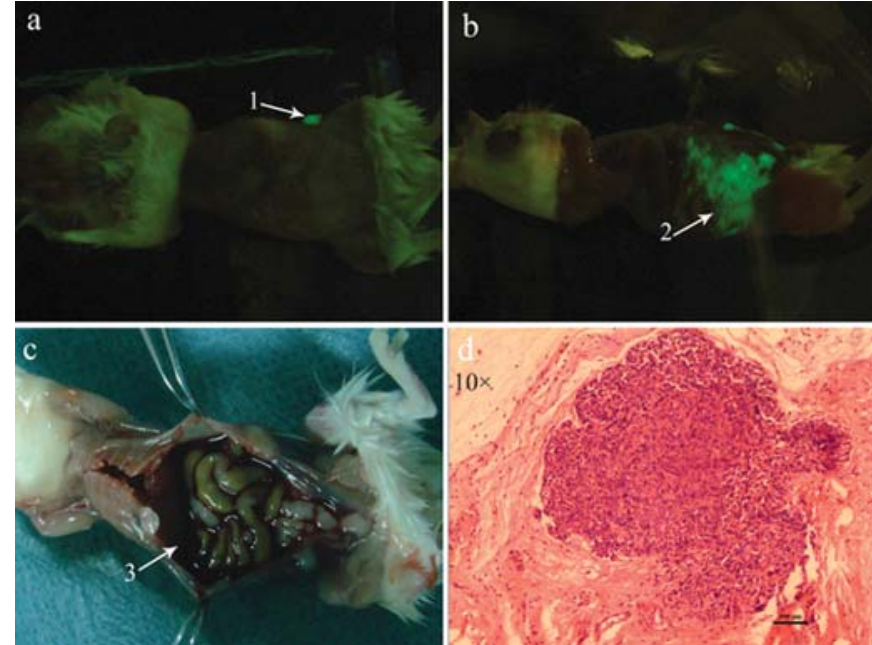

Figure 4. Gross observation and histological examination of orthotopic and metastatic tumors of TM40D in the 'breast-breast' and 'MFP-breast' model groups. TM40D grew better in orthotopic MFP than in human breast tissue. These cells metastasized to the mouse viscera much more widely when the MFP of mice were inoculated compared to when implanted human breast tissues were inoculated. (a) Fluorescent gross observation of the 'breastbreast' model (dorsal position) with TM40D. (b) Fluorescent gross observation of the 'MFP-breast' model (dorsal position) with TM40D. (c) Gross observation of the 'MFP-breast' model (dorsal position) with TM40D. (d) H\&E staining of orthotopic tumors in human breast tissue of the 'breast-breast' model with TM40D. 1, Fluorescent orthotopic tumors in implanted human breast tissue; 2 , fluorescent metastatic tumors in abdominal viscera; 3 , severe bloody ascites in the 'MFP-breast' model with TM40D. Scale, $100 \mu \mathrm{m}$ (d). Magnification of objective lens, $\mathrm{x} 10$.

(Table III, Fig. 3a, b, e and f). In the MPF-bone model, MDA-MB-231BO cells formed visible tumors in all orthotopic MFP mice (9 of 9), while MDA-MB-231 cells formed no visible tumors ( 0 of 10 ) (Table IV).

We also noted frequent involvement of the mesentery in mouse tissue in both the BOM ('breast-breast', 'breastbone') and the MFP ('MFP-bone', 'MFP-breast') models $(31 / 62)$ but not in the 'bone-bone' model (0/19), while mouse bone, lung, liver, and brain were rarely involved. Compared with the 'breast-breast' and the MFP-breast model, the mouse breast cell line TM40D grew better in orthotopic MFP than in human breast tissue. Furthermore, these cells metastasized to the mouse viscera much more widely when the MFP of mice were inoculated compared to when implanted human breast tissues were inoculated (Table V, Fig. 4). Results from these groups above showed that tumorigenicity was weaker in human tissue and moderately enhanced in mouse tissue following inoculation of breast cancer cells into human breast tissue, around human bone (combined with human bone and the mouse microenvironment) and into mouse MFP (Table VI).

\section{Discussion}

In our previous BOM model ('breast-breast' model), both the orthotopic and metastatic sites contained breast cancer cells of human origin. In addition, the dissemination of breast cancer cells was repeated and slight (10). All of these unique features of the BOM model mimic the clinical situation and thus, significantly distinguish this model from other mouse 
Table III. Tumor formation of MDA-MB-231BO compared with MDA-MB-231 in 'bone-bone' model.

\begin{tabular}{lccccccc}
\hline Bo-Bo & Lf-Bo & Rt-Bo & Ms-Bo & Mes & Ms-Lv & Ms-Lu & Bl-as \\
\hline 231 BO & $6 / 7$ & $0 / 7$ & $0 / 7$ & $0 / 7$ & $0 / 7$ & $0 / 7$ & $0 / 7$ \\
231 & $9 / 12$ & $0 / 12$ & $0 / 12$ & $0 / 12$ & $0 / 12$ & $0 / 12$ & $0 / 12$ \\
\hline
\end{tabular}

MDA-MB-231BO (6/7) and MDA-MB-231 (9/12) were detected in implanted human bone in 'bone-bone' model. They could not metastasize to distant human and mouse bone. N/M, breast cancer cells were detected in corresponding tissue in $\mathrm{N}$ of $\mathrm{M}$ mice. Bo-Bo, 'bone-bone' model; Lf-Bo, implanted human bone in left flank (inoculating site); Rt-Bo, implanted human bone in right flank; Ms-Bo, mouse bone; Mes, mouse mesentery; Ms-Lv, mouse liver; Ms-Lu, mouse lung. Bl-as, bloody ascites; 231BO, MDA-MB-231BO; 231, MDA-MB-231.

Table IV. Tumor formation of MDA-MB-231BO compared with MDA-MB-231 in 'MFP-bone' model.

\begin{tabular}{lccccccc}
\hline MFP-Bo & Lf-MFP & Rt-Bo & Ms-Bo & Mes & Ms-Lv & Ms-Lu & Bl-as \\
\hline $231 \mathrm{BO}$ & $9 / 9^{\mathrm{a}}$ & $0 / 9$ & $0 / 9$ & $4 / 9$ & $1 / 9$ & $0 / 9$ & $0 / 9$ \\
231 & $0 / 10$ & $0 / 10$ & $0 / 10$ & $8 / 10$ & $0 / 10$ & $0 / 10$ & $0 / 10$ \\
\hline
\end{tabular}

MDA-MB-231BO formed visible tumors in orthotopic site of all mice (9/9). MDA-MB-231 failed to form visible tumor in the same site $(0 / 10)$. They could not metastasize to distant human and mouse bone. N/M, breast cancer cells were detected in corresponding tissue in $\mathrm{N}$ of M mice. MFP-Bo, 'MFP-breast' model. Lf-MFP, mouse mammary fat pad near left inguina (inoculating site); Rt-Bo, implanted human bone in right flank; Ms-Bo, mouse bone; Mes, mouse mesentery; Ms-Lv, mouse liver; Ms-Lu, mouse lung. Bl-as, bloody ascites; 231BO, MDA-MB-231BO; 231, MDA-MB-231. ${ }^{\mathrm{a}} \mathrm{p}<0.05$, MDA-MB-231BO compared with MDA-MB-231.

Table V. Tumor formation of TM40D in 'breast-breast' model compared with 'MFP-breast' model.

\begin{tabular}{lcccccc}
\hline TM40D & Lf-Br/MFP & Rt-Br & Ms-Bo & Mes & Ms-Lv & Ms-Lu \\
\hline Br-Br & $6 / 8$ & $0 / 8$ & $0 / 8$ & $0 / 8$ & $0 / 8$ & $1 / 8$ \\
MFP-Br & $8 / 8$ & $0 / 8$ & $0 / 8$ & $8 / 8^{\mathrm{a}}$ & $2 / 8$ & $1 / 8$ \\
\hline
\end{tabular}

Mouse breast cancer cells TM40D grew and metastasized much more fiercely in 'MFP-breast' model than in 'breast-breast' model. N/M, breast cancer cells were detected in corresponding tissue in $\mathrm{N}$ of $\mathrm{M}$ mice. Br-Br, 'breast-breast' model; MFP-Br, 'MFP-breast' model; Lf-MFP, mouse mammary fat pad near left inguina (inoculating site); Lf-Br, implanted human breast tissue in left flank (inoculating site); Rt-Br, implanted human breast tissue in right flank; Ms-Bo, mouse bone; Mes, mouse mesentery; Ms-Lv, mouse liver; Ms-Lu, mouse lung. Bl-as, bloody ascites. ${ }^{\mathrm{a}} \mathrm{p}<0.05$, 'MFP-breast' model compared with 'breast-breast' model.

models. It is well known that bone is the most frequent metastatic site of breast cancer, and bone metastasis comprises the most significant tumor burden in patients (18). In the onset of this study, we established a novel BOM model consisting of human breast tissue implantation and human bone implantation into the left and right flanks of SCID mice, respectively, to mimic the clinical situation of breast cancer bone metastasis. Using MDA-MB-231 cells, tumors could be detected in implanted human breast tissue but not in human bone. Furthermore, no mouse bone metastasis was detected, which differed from widely used traditional mouse models.

Based on morphological, immunohistochemical, and enzymohistochemical results, the adult female human bone fragments implanted into SCID mice were both viable and functional for at least 12 weeks post-transplantation. All bone cell types (osteoblasts, osteoclasts, osteocytes, adipocytes, and stromal cells) were present and apparently viable. The implanted human bone was functional, as determined by high levels of human immunoglobulin ( $\mathrm{IgG}$ ) and the presence of circulating human B cells in the mouse circulation. Human CD34 expression on vascular endothelial cells indicated that human blood vessels were also present in the implanted bone. In addition, extensive neovascularization occurred to supply blood to the implants. All these suggested that the implanted human breast tissue was viable and functional. The normal human breast tissues continued to reserve the 
Table VI. Metastatic conditions of MDA-MB-231 cells and MDA-MB-231BO cells inoculated into different orthotopic microenvironments.

\begin{tabular}{lccccc}
\hline & \multicolumn{4}{c}{ Cancer cell detected } \\
\cline { 2 - 3 } & \multicolumn{2}{c}{231} & & \multicolumn{2}{c}{$231 \mathrm{BO}$} \\
\cline { 2 - 3 } \cline { 5 - 6 } $\begin{array}{l}\text { Inoculating } \\
\text { cite }\end{array}$ & Hu-exc & Mou-exc & Hu-exc & Mou-exc \\
\hline Hu-Br & $11 / 18$ & $0 / 18$ & & $6 / 9^{\mathrm{a}}$ & $0 / 9$ \\
Hu-Bo & $5 / 12$ & $3 / 12$ & & $1 / 7$ & $1 / 7$ \\
Mou-MFP & - & $8 / 10^{\mathrm{b}}$ & & $5 / 9^{\mathrm{b}}$ \\
\hline
\end{tabular}

Tumorigenicity was weaker in human tissue and moderately enhanced in mouse tissue following inoculation of breast cancer cells into human breast tissue, around human bone and into mouse MFP. Hu-br, implanted human breast tissue; Hu-bo, implanted human bone; Mou-MFP, inoculated mammary fat pad of mouse; Hu-exc, mice with tumor in implanted human tissue but any metastasis in mouse tissue; Mou-exc, mice with metastasis in mouse tissue exclusively. ${ }^{\mathrm{a}} \mathrm{p}<0.05$, Hu-br group compared with Hu-bo group. ${ }^{\mathrm{b}} \mathrm{p}<0.05$, Mou-MFP group compared with $\mathrm{Hu}$-bo group.

glandular tube and/or lobular architecture, as well as a wide variety of the normal breast cell types, such as the breast epithelial cells and the adipocytes. The human-derived vessels remained viable within the implants for a period of 9 weeks after implantation suggesting that the implanted human breast tissue was viable during the entire in vivo period.

The breast cancer cell line MDA-MB-231 was first isolated from a pleural effusion from a patient with disseminated disease relapsing several years after removal of the primary tumor (19). In intracardiac/tail-vein inoculated immunodeficient mouse models, these cells formed mouse bone metastases frequently, which initiated the use of these cells as an osteotropic cell line in many studies $(14,20,21)$. Some studies, however, indicated that the method of injection influenced the metastatic tropism $(7,14,22-24)$. Single-cell progenies (SCP) of MDA-MB-231 also showed a similar poor prognosis signature, but varied in metastatic tropism, and exhibited osteotropic, other organ-tropic, single-organ tropic, or multi-organ tropic properties (25). Although MDAMB-231 cells could form bone metastases in some specified conditions, such as following injection into the circulation or injection directly into bone, these cells did not exhibit any osteotropism in human microenvironments in this study. Since the implanted orthotopic site, the metastatic site, and the pathway between the sites were available, specific properties of MDA-MB-231 cells might explain the absence of bone metastasis. Later, a well-known osteotropic subclone of MDA-MB-231 cells, MDA-MB-231BO, was introduced to the BOM ('breast-bone') model. Surprisingly, similarly to MDA-MB-231 cells, no metastasis was detected in implanted human bone, both macroscopically and microscopically.

The 'breast-bone' model and the 'breast-breast' model were identical for the orthotopic site and cancer cell type, but differed in the metastatic site. To exclude any unknown factors that potentially interfere with breast cancer cell proliferation that might be introduced by the implantation process, a 'bone-bone' model was used in which human bone was implanted into both left and right flanks subcutaneously, and breast cancer cells were inoculated around the left implanted bone. Visible tumor masses formed in inoculated human bone, but no cancer cells were detected in the opposite flank containing human bone both macroscopically and microscopically. In addition, there was no significant difference between MDA-MB-231BO cells and MDA-MB-231 cells in the 'bone-bone' model. Considerable metastases formed in human breast tissue in the metastatic site of the BOM ('breast-breast') model, indicating that neither MDA-MB-231 cells nor the osteotropic subclone MDA-MB-231BO, which was established based on the intracardiac mouse model by repeated sequential passages in nude mice and in vitro of metastatic cells obtained from bone metastases, were osteotropic.

We noted that both MDA-MB-231BO cells and MDAMB-231 cells failed to form any mouse bone metastases in the 'breast-breast' and the 'breast-bone' models, which differed from most traditional circulation-inoculation mouse models. It is well known that circulating tumor cells (DTCs) in breast cancer patients are rarely detectable. There are usually only 10 breast cancer cells per one million bone marrow cells, and DTCs are not detectable in every patient, even in those who develop relapsing breast cancer $(26,27)$. The bone-metastasis formation in the circulation-inoculation mouse model does not imply osteotropism of breast cancer cells. Those models failed to encompass the molecular mechanisms by which breast cancer cells are attracted to target organs in a clinical situation. The bone metastasis can be attributed to the interaction between the great number of cancer cells and the capillary bed first encountered when cancer cells are injected directly into the mouse circulation, rather than the tropism of the cancer cell itself (28). To confirm these results, the 'MFP-bone' model was used. Neither human bone nor mouse bone was metastastically involved following inoculation with MDA-MB-231BO or MDA-MB-231 cells.

Additionally, compared to MDA-MB-231 cells, when inoculated into human breast tissue and MFP mice, MDAMB-231BO cells displayed higher tumorigenicity, but no difference in osteotropism. These cells formed significantly larger masses in human breast tissue, and formed visible tumors in MFP mice; MDA-MB-231 cells failed to do so. These results indicated that the repeat passages in mice that were adverse to human cells enhanced the poordiagnosis feature rather than selecting for the organ-specific tropism. These data were consistent with the SCP study of MDA-MB-231 cells (25). The unsuitable heterogeneic microenvironment in the mouse enhanced the adaptability, tumorigenicity, and invasion of these cells, while the organspecific tropism was not well developed because of the species disparity.

In studies based on mouse models, interactions between breast cancer cells and the microenvironment were essential (29-32). When cells were inoculated into human breast, around human bone (combined with human bone and the 
mouse microenvironment) and into mouse MFP, the tumorigenicity was weakened in human tissue, but moderately enhanced in mouse tissue. In BOM models, MFP models, and the 'bone-bone' model, the frequent target organs mentioned in traditional mouse models including bone, lung, liver, and brain were rarely involved, while mesentery was involved generally and frequently. The behavior pattern of breast cancer cells in the mouse model is mainly regulated by two types of interactions: the species-specific interactions and the organ-specific interactions between cancer cells and host tissue. To confirm these observations, the mouse breast cancer cell line TM40D was introduced to the 'breast-breast' model and the 'MFP-breast' model. These cells failed to form any metastases in the opposing flank containing human breast tissue in the two models. In the 'breast-breast' model, TM40D cells grew in human breast tissue in the inoculating site and rarely metastasized to mouse tissue. In the 'MFPbreast' model, these cells grew and metastasized in mouse tissues vigorously, which induced bloody ascites in seven of eight mice in this group and caused death for two mice before the end point of the experiment.

Multiple comparative in vivo experiments in the present study proved BOM models were feasible and reliable by confirming every general aspect of breast cancer bone metastasis. The differences in the metastatic phenotype of MDA-MB231 cells between the BOM model and other mouse models without a human microenvironment suggested that the true metastatic phenotype can not be ascertained through the method of blood circulation inoculation. Species-specific and tissue-specific interactions between breast cancer cells and host tissues influence the behavior of tumors in orthotopic and metastatic mouse models. The human tissue-specific microenvironment is a fundamental factor in orthotopic and metastatic breast cancer mouse models of clinically relevant studies. Further investigation is required to complete the breast cancer metastatic variant theory and practice, such as the isolation of subclones based on the BOM model principle. Studies regarding metastatic variants of breast cancer should consider human tissue-specific microenvironment as an essential factor. At the very least, conclusions should be confirmed by reasonable models with human tissue-specific microenvironments and the most clinically relevant inoculation methods. These principles may also be suitable for mouse models of many other solid tumors.

\section{Acknowledgments}

We are grateful to Dr Bei-Cheng Sun (Center of Liver Transplantation, the First Affiliated Hospital of Nanjing Medical University) for providing us with pLenti-GFP lentivirus, to Dr Jie Wang (Department of Medical Research, Guangzhou General Hospital of Guangzhou Command) for providing us with MDA-MB-231BO cells, and to Dr Ji-Fu Wei (Clinical Experiment Center, the First Affiliated Hospital of Nanjing Medical University) for critical discussion of our study. This research was supported in part by the National Natural Science Foundation of China (30740076), the Six Kinds of Outstanding Talent Foundation of Jiangsu Province (06-B-069), the Science and Education for Health foundation of Jiangsu Province (RC2007054), and the Natural
Science Foundation of Jiangsu Province (BK2008476 and BK2009438).

\section{References}

1. Jemal A, Siegel R, Ward E, Hao Y, Xu J and Thun MJ: Cancer statistics, 2009. CA Cancer J Clin 59: 225-249, 2009.

2. Sharpless NE and Depinho RA: The mighty mouse: genetically engineered mouse models in cancer drug development. Nat Rev Drug Discov 5: 741-754, 2006.

3. Kelland LR: Of mice and men: values and liabilities of the athymic nude mouse model in anticancer drug development. Eur J Cancer 40: 827-836, 2004.

4. Kamb A: What's wrong with our cancer models? Nat Rev Drug Discov 4: 161-165, 2005.

5. Kung AL: Practices and pitfalls of mouse cancer models in drug discovery. Adv Cancer Res 96: 191-212, 2007.

6. Kerbel RS: Human tumor xenografts as predictive preclinical models for anticancer drug activity in humans: better than commonly perceived-but they can be improved. Cancer Biol Ther 2: 134-139, 2003.

7. Sausville EA and Burger AM: Contributions of human tumor xenografts to anticancer drug development. Cancer Res 66: 3351-3354, 2006

8. Hoffman RM: Orthotopic metastatic (MetaMouse) models for discovery and development of novel chemotherapy. Methods Mol Med 111: 297-322, 2005.

9. Inaba M, Tashiro T, Kobayashi T, et al: Responsiveness of human gastric tumors implanted in nude mice to clinically equivalent doses of various antitumor agents. Jpn J Cancer Res 79: 517-522, 1988.

10. Wang J, Xia TS, Liu XA, et al: A novel orthotopic and metastatic mouse model of breast cancer in human mammary microenvironment. Breast Cancer Res Treat 120: 337-344, 2010.

11. Chambers AF, Groom AC and MacDonald IC: Dissemination and growth of cancer cells in metastatic sites. Nat Rev Cancer 2: 563-572, 2002 .

12. Fidler IJ: The pathogenesis of cancer metastasis: the 'seed and soil' hypothesis revisited. Nat Rev Cancer 3: 453-458, 2003.

13. Yoneda T, Williams PJ, Hiraga T, Niewolna M and Nishimura R: A bone-seeking clone exhibits different biological properties from the MDA-MB-231 parental human breast cancer cells and a brain-seeking clone in vivo and in vitro. $J$ Bone Miner Res 16: 1486-1495, 2001.

14. Garcia T, Jackson A, Bachelier R, et al: A convenient clinically relevant model of human breast cancer bone metastasis. Clin Exp Metastasis 25: 33-42, 2008.

15. Laidlaw IJ, Clarke RB, Howell A, Owen AW, Potten CS and Anderson E: The proliferation of normal human breast tissue implanted into athymic nude mice is stimulated by estrogen but not progesterone. Endocrinology 136: 164-171, 1995.

16. Nemeth JA, Harb JF, Barroso U Jr, He Z, Grignon DJ and Cher ML: Severe combined immunodeficient-hu model of human prostate cancer metastasis to human bone. Cancer Res 59: 1987-1993, 1999.

17. Yonou H, Yokose T, Kamijo T, et al: Establishment of a novel species- and tissue-specific metastasis model of human prostate cancer in humanized non-obese diabetic/severe combined immuno-deficient mice engrafted with human adult lung and bone. Cancer Res 61: 2177-2182, 2001.

18. Mundy GR: Metastasis to bone: causes, consequences and therapeutic opportunities. Nat Rev Cancer 2: 584-593, 2002.

19. Cailleau R, Young R, Olivé M and Reeves WJ Jr: Breast tumor cell lines from pleural effusions. J Natl Cancer Inst 53: 661-674, 1974.

20. Pratap J, Wixted JJ, Gaur T, et al: Runx2 transcriptional activation of Indian Hedgehog and a downstream bone metastatic pathway in breast cancer cells. Cancer Res 68: 77957802,2008 .

21. Neudert M, Fischer C, Krempien B, Seibel MJ and Bauss F: A rapid histological score for the semiquantitative assessment of bone metastases in experimental models of breast cancer. Onkologie 31: 521-527, 2008.

22. Miner KM, Kawaguchi T, Uba GW and Nicolson GL: Clonal drift of cell surface, melanogenic, and experimental metastatic properties of in vivo-selected, brain meninges-colonizing murine B16 melanoma. Cancer Res 42: 4631-4638, 1982. 
23. Gotzmann J, Mikula M, Eger A, et al: Molecular aspects of epithelial cell plasticity: implications for local tumor invasion and metastasis. Mutat Res 566: 9-20, 2004.

24. Nicolson GL, Belloni PN, Tressler RJ, Dulski K, Inoue T and Cavanaugh PG: Adhesive, invasive, and growth properties of selected metastatic variants of a murine large-cell lymphoma. Invasion Metastasis 9: 102-116, 1989.

25. Minn AJ, Kang Y, Serganova I, et al: Distinct organ-specific metastatic potential of individual breast cancer cells and primary tumors. J Clin Invest 115: 44-55, 2005.

26. Gilbey AM, Burnett D, Coleman RE and Holen I: The detection of circulating breast cancer cells in blood. J Clin Pathol 57: 903-911, 2004

27. Riethdorf S and Pantel K: Disseminated tumor cells in bone marrow and circulating tumor cells in blood of breast cancer patients: current state of detection and characterization. Pathobiology 75: 140-148, 2008

28. Ottewell PD, Coleman RE and Holen I: From genetic abnormality to metastases: murine models of breast cancer and their use in the development of anticancer therapies. Breast Cancer Res Treat 96: 101-113, 2006.
29. Ottewell PD, Deux B, Mönkkönen H, et al: Differential effect of doxorubicin and zoledronic acid on intraosseous versus extraosseous breast tumor growth in vivo. Clin Cancer Res 14: 4658-4666, 2008.

30. Winding B, Misander H, Høegh-Andersen P, Brünner N and Foged NT: Estradiol enhances osteolytic lesions in mice inoculated with human estrogen receptor-negative MDA-231 breast cancer cells in vivo. Breast Cancer Res Treat 78: 205-216, 2003.

31. Kuperwasser C, Dessain S, Bierbaum BE, et al: A mouse model of human breast cancer metastasis to human bone. Cancer Res 65: 6130-6138, 2005.

32. Yang W, Lam P, Kitching R, et al: Breast cancer metastasis in a human bone NOD/SCID mouse model. Cancer Biol Ther 6: 1289-1294, 2007. 